\title{
Application of the Method of Means to the Stability Analysis of Unbraced Frames
}

\author{
Jostein Hellesland \\ Professor, Mechanics Division, Department of Mathematics, \\ University of Oslo, P. O. Box 1053 - Blindern, 0316 Oslo, Norway
}

\begin{abstract}
Approximate methods for determination of critical loads and effective lengths of compression members in frame systems are reviewed, discussed and applied to selected unbraced low-rise and high-rise multistorey frames. The methods make use of effective lengths from isolated column analyses to arrive at improved values. They satisfy general system instability principles, are rather simple, and yield in general effective length predictions in good agreement with exact results for a wide variation of parameters. Main emphasis is on a method previously presented and denoted the method of means. Extensions of the method pertinent to unbraced multistorey frames are presented.
\end{abstract}

\section{KEYWORDS}

Analysis, Structures, Stability, Buckling, Columns, Compression Members, Effective Length, Critical Load, Frames, Storeys 


\section{INTRODUCTION}

In design of structures that include slender compression members, it is necessary to consider stability and second order load effects. For unbraced frames, design codes generally allow such evaluations to be based on approximate storey-based approaches. Such methods normally reflect the horizontal interaction between the columns of a storey quite well, and also the vertical interaction between storeys, provided the columns exhibit inflection points in the vicinity of their midheights. When this is not the case, such as in the lower storey(s) of multistorey frames with stiff columns and flexible beams, for which the first inflection point above the base may be located near the top of the bottom storey or several storeys up, the predictions of critical loads by isolated column considerations or standard storey-based approaches may become quite inaccurate.

The paper considers such, and other examples, reviews some existing methods for critical load and effective length calculation and demonstrates how a rather simple method, denoted the "method of means" (Hellesland and Bjorhovde 1996b), can be applied to improve predictions. The method, including an alternative application (Hellesland 1995), is briefly reviewed and extensions relevant to larger frameworks are presented.

\section{THE METHOD OF MEANS}

The critical loading at which a structure, with a given initial axial force $(P)$ distribution in the members, is on the verge of instability (buckling), can be defined through a single (critical) load factor $\gamma_{c r}$ in the common case of proportional loading. Inversely, it may be defined through a single system stability index, $\alpha_{\text {system }}=1 / \gamma_{\mathrm{cr}}$, common to all members.

Alternatively, the critical loading may be reflected in terms of the effective length factor of any individual compression member. Neglecting shear deformations, the elastic critical load $P_{\text {cr }}$ and effective length factor $K$ of a compression member (column, strut, etc.) of length $L$ and sectional bending stiffness $E I$ are defined by

$$
P_{\mathrm{cr}}=\frac{\pi^{2} E I}{(K L)^{2}} \quad \text { and } \quad K=\sqrt{\frac{P_{E}}{P_{\mathrm{cr}}}}=\sqrt{\frac{\alpha_{\text {system }}}{\alpha_{E}}}
$$

where

$$
\alpha_{E}=\frac{P}{P_{E}} \quad \text { and } \quad P_{E}=\frac{\pi^{2} E I}{L^{2}}
$$

is a nominal member flexibility parameter ("load parameter") and the Euler buckling load of a pin-ended member, respectively. Above, EI and $P$ are the values at a chosen reference section for members with varying stiffness and axial force along the length. The relationship between the effective length factors of any two members, $i$ and $j$, are interrelated through the common stability index 
and becomes

$$
K_{i}=K_{j} \sqrt{Q_{j i}}
$$

where

$$
Q_{j i}=\frac{\alpha_{E j}}{\alpha_{E i}}=\frac{P_{j}}{P_{i}} \frac{L_{j}^{2}}{L_{i}^{2}} \frac{E I_{i}}{E I_{j}}=\frac{(P L)_{j}}{(P L)_{i}} \frac{(E I / L)_{i}}{(E I / L)_{j}}
$$

is the members' nominal flexibilities ratio. The first subscript in $Q$ refers to $\alpha_{E}$ in the numerator, and the second to $\alpha_{E}$ in the denominator. Thus, inverse values are obtained by reversing subscripts.

In approximate methods, the stability analysis may commence by computing approximate $K$-factors of the various compression members considered in isolation. For each compression member $(i)$ a corresponding isolated member stability index

$$
\alpha_{i}=\left(P / P_{\mathrm{cr}}\right)_{i}=\left(K^{2} \alpha_{E}\right)_{i}
$$

can be computed. In a frame having members with different flexibilities, these indices will generally not become equal due to approximations in the end restraint assessments. Thus, such isolated predictions violate basic system instability requirements. However, these pseudo member stability indices, and the pseudo effective length factors, may be used as parameters in procedures that may yield improved results. For "isolated" $K$-factor predictions, many, and possibly most, national and international design codes and standards give rotational end restraints, in one form or the other, as implied from "conventional" joint stiffness ratios given by

$$
G_{J}=\frac{\left(\sum E I / L\right)_{J}}{\left(\sum m E I_{b} / L_{b}\right)_{J}} \quad J=A, B
$$

Here the summations are over the compression members (numerator) and flexural members (denominator; subscript $b$ ) meeting at the joint $(A$ or $B$ ), and $m$ is a stiffness modifier that is equal to unity in the datum cases of rigidly connected and 1) symmetrically bent flexural members restraining braced compression members and 2) antisymmetrically bent flexural members restraining unbraced compression members.

Based on such $G$-factors, effective lengths tend to be underestimated in the stiffer compression members and overestimated in the more flexible members. This is so for several reasons, including an incorrect reflection in the $G$-factor definition of the (vertical) interaction between compression members at a joint (Hellesland and Bjorhovde 1996a).

In the method of means (MOM), an average of all or a certain selection of compression member indices may be adopted as an approximation of the system stability index.Thus,

$$
\alpha_{\text {system }}=\bar{\alpha}=\frac{1}{m} \sum^{m} \alpha_{i}
$$


where $m$ is the number of interacting members considered. A "mean" effective length factor for an arbitrary member $j$ can then be obtained in terms of $\bar{\alpha}$ or isolated $K$-values from

$$
\bar{K}_{j}=\sqrt{\frac{\bar{\alpha}}{\alpha_{E j}}}=\left[\frac{1}{m} \sum^{m} K_{i}^{2} Q_{i j}\right]^{1 / 2}
$$

Once the mean $\bar{K}_{j}$-value is computed for a chosen reference member $j$, the corresponding mean values for the other members can alternatively be computed from this value using Eq. (3).

If the $\bar{K}$-prediction for a member becomes less than some known or estimated lower limit, advantage should be taken of this knowledge to improve $\bar{K}$-predictions. For further discussion, see Hellesland and Bjorhovde (1996b), Hellesland (1997) and the discussion of multistorey frames below. If a lower limit governs member $k\left(\lim \bar{K}_{k}>\bar{K}_{k}\right)$, then improved effective lengths become

$$
\bar{K}_{i}=\left(\lim \bar{K}_{k}\right) \sqrt{Q_{k i}} \quad i=1,2, \ldots
$$

The calculations of Eq. (9) should be carried out based on the member $(k)$ that yields the larger $\bar{K}_{i}$-values. This will generally be the member with the largest term (member stability index) in the summation. Eq. (9) corresponds to $\alpha_{\text {system }}=$ $\left(\lim \bar{K}_{k}\right)^{2} \alpha_{E k}$.

It may be a matter of judgment which compression members to include in the summation of Eq. (7). In previous applications to braced and unbraced single or low rise multistorey frames, all columns were included (Hellesland and Bjorhovde 1996b; Hellesland 1995, 1997). However, for large multistorey frameworks it has been suggested (Hellesland and Bjorhovde 1996b) that a partial application to a limited number of interacting columns in a limited region of a frame may be adequate. Probably, it is not only adequate, but also preferable in order not to "suppress" localized failure in a region that may be significantly more flexible than the rest of the structure. For braced structures, a partial application to a limited number of interconnected compression members is probably most practical and appropriate. For unbraced multistorey frames, a practical approach is proposed in this paper. Also, alternative stability indices that may be adopted in conjunction with the method of means for unbraced frames (Hellesland 1995) are reviewed below.

\section{BRACED BUCKLING}

For unbraced (sway permitted) frames, the lowest critical load will be due to buckling in the lateral sway mode provided none of the columns in a storey are so flexible relative to the others in the storey as to inflict "premature" instability by local buckling between column ends, i.e. in an essentially braced mode. In 
approximate methods, these two modes of failure will normally have to be checked independently.

Local, "braced" buckling may be checked in an approximate manner by first considering the frame to be fully braced. The system stability index obtained for the braced frame may then be increased by $10 \%$ or so to reflect some sway action. This is generally found to give acceptable results. A conservative (safe) estimate for the braced frame is given by the largest of the various member stability indices, Eq. (5), obtained on the basis of isolated $K$-factors for braced columns. A similar provision for braced frames is given in AS4100 (Australian Standard 1990) and an indication of the same in Eurocode 3 (1992). A less conservative estimate, in particular for frames with beams that are relatively flexible compared to the columns, can be obtained by the method of means.

\section{SWAY BUCKLING - ISOLATED STOREY}

The lateral (horizontal) interaction between columns of various stiffness in a storey of an unbraced frame, resulting from the common translational displacement $(\Delta)$ of the top relative to the base of the columns, is well understood. As an alternative to approaches presented below, a storey sway stability index may be established by the method of means as

$$
\alpha_{\text {storey }}=\bar{\alpha}=\frac{1}{m} \sum^{m} \alpha_{i}
$$

where $m$ is the total number of interacting columns in the storey and $\alpha_{i}$ the member stability indices (Eq. (5)) based on isolated, free-to-sway $K$-factors of the columns. Eq. (10) is not applicable to unbraced frames that include pinended columns (due to the infinite isolated $K$-factors of such columns). Also, Eq. (10) may give very conservative estimates for unbraced frames that include columns that otherwise are very flexible compared to the others. Suggested limits of applicability are given in Hellesland and Bjorhovde (1996b). In a modified form (Hellesland 1995), it may be applied beyond these limits as well as to frames with pin-ended columns.

More physically based predictions reflecting the storey response may be derived by methods that explicitly consider the $P \Delta$ effects. These yield sway magnification factors such as $f_{s}=1 /\left(1-\alpha_{s}\right)$, where $\alpha_{s}$ is a storey sway stability index of the types derived for instance by Hellesland (1976),

$$
\alpha_{s \Delta}=\frac{\sum \gamma P / L}{H / \Delta_{o H}} \quad \text { or } \quad \alpha_{s}=\frac{\sum \gamma P / L}{\sum \gamma P_{\mathrm{cr}} / L}
$$

The summations are over all columns in the storey, the ratio $H / \Delta_{o H}$ is the first order lateral storey stiffness (lateral load (sum of shears) per unit lateral dis- 
placement of the top relative to the base of the columns) and $P_{\text {cr }}$ (Eq. (1)) is to be based on the columns' isolated (free-to-sway) $K$-factors. With equal column lengths and with $\gamma=1.0$, these stability indices reduce to the more well-known forms adopted in one form or the other by a number of design codes (ACI, AISC, AS4100, Eurocode 3, etc.).

Due to axial force effects, the moment distribution along the columns become nonlinear and the lateral column stiffnesses become less than their first order values. This effect is accounted for by the "flexibility" factor $\gamma$. When evaluated at the free sway (zero shear) condition, it takes on values between 1 and 1.22 for columns with positive end restraints. A simple, yet rather accurate, expression for $\gamma$ is given by

$$
\gamma=1+0.11 \frac{1+\left[1-\left(0.5 G_{\max }\right)^{p}\right]^{3}}{\left(1+0.5 G_{\min }\right)^{2}}
$$

where $p=1$ for $G_{\max } \leq 2$ and $p=-1$ for $G_{\max }>2$. $G_{\max }$ is the larger and $G_{\min }$ the smaller of the $G$-factors at the column ends. This expression was proposed by the author in 1981 (during a research stay at the University of Alberta, Edmonton). For a column pinned at one end, it breaks down to an expression derived earlier by Hellesland (1976) and, in a different form $\left(C_{L}=\right.$ $1-\gamma$ ), by LeMessurier (1977).

The effective length factor for a column $j$ in a single storey frame can now be expressed in terms of either one of the expressions above. For instance, in terms of $\bar{\alpha}$ by Eq. (8) and in terms of $\alpha_{s}$, in the common case with constant column lengths, by

$$
K_{s j}=\sqrt{\frac{\alpha_{s}}{\alpha_{E j}}}=\left[\frac{E I_{j}}{P_{j}} \frac{\sum \gamma P}{\sum \gamma E I / K^{2}}\right]^{1 / 2}
$$

where the subscript $s$ is added for the sake of distinction. The $\gamma$-factor for the various columns will often be nearly equal, and can normally be deleted. This expression is very similar to one given by LeMessurier (1977) and gives identical results for the same values of $\gamma$.

\section{SWAY BUCKLING - MULTISTOREY FRAMES}

For multistorey sway frames, the storey sway stability indices predicted for the various storeys using any of the expressions given above, will generally become different for the different storeys. Therefore, the predictions will not satisfy basic system instability requirements.

An approximate, conservative estimate of the system stability index can be expected to be given by the largest of the storey stability indices of the various storeys. Thus,

$$
\alpha_{\text {system }}=\max \left(\alpha_{\text {storey }, i}\right)
$$


where $\alpha_{\text {storey }}$ may be based on $\alpha_{s \Delta}, \alpha_{s}$ or $\bar{\alpha}$.

When $\alpha_{s \Delta}$ is used, and the first order lateral storey stiffnesses $\left(H / \Delta_{o H}\right)$ are those obtained on the basis of a first order linear elastic analysis of the frame subjected to a specific lateral load pattern, Horne (1975) has shown that the estimate is on the safe side and always within $20 \%$ of the correct result. Thus, effective lengths (Eq. (2)) will be within 10\%. The load pattern adopted by Horne consisted of concentrated horizontal loads applied at each storey level and taken as some proportion (the same at each level) of the vertical loads on that level. The frames considered had prismatic members and $\gamma=1 / 0.9=1.11$ was adopted for all columns. With $\gamma=1.0$, the maximum error is reduced from 20 to about $10 \%$, but the estimate may not longer be on the safe side.

Eq. (14) based on $\alpha_{s}$ with $\gamma=1$, is similar to a system instability condition given in AS4100 (Australian Standard 1990) for rectangular frames. Whether based on $\alpha_{s}$ or $\bar{\alpha}$, it will generally provide good estimates when the governing storey is separated from the adjacent storeys by beams that are relatively stiff compared to the columns of the storey. However, in cases with strong interaction between columns in adjacent storeys (flexible beams and stiff columns), Eq. (14) may give very conservative estimates.

An improved approximation of system instability may in such cases be obtained by applying the method of means to the storey stability indices $\alpha_{s i}$ or $\bar{\alpha}_{i}$. Unlike $\alpha_{s \Delta i}$, these tend to be underestimated in the stiffer storeys and overestimated in the more flexible storeys. For low rise frames it is appropriate to include all storeys in the calculation of the mean. For multistorey frames, a partial application of the method to a limited number of adjacent storeys in the most flexible region of the frame may be more appropriate. By including distant storeys, with negligible or weak interaction with the critical (most flexible) region, a localized failure may be "suppressed" and the system stability index underestimated. From a study of a number of frames, it seems that the local, "regional" mean of the most flexible region may be representative.

An approximate system stability index for sway buckling, that reflects aspects discussed above, can be obtained on the basis of storey stability indices $\alpha_{s i}$ (or $\left.\bar{\alpha}_{i}\right)$ from

$$
\alpha_{\text {system }}=\bar{\alpha}_{s}=\frac{1}{n} \sum^{n} \alpha_{s i}
$$

where the summation should be taken over a limited number $n$ of consecutive storeys such as to reflect the interaction between the storeys and to provide a maximum local mean. Normally it should be taken over the storey with the largest storey stability index and the adjacent storey above and below, and as many additional consecutive storeys to either side that are necessary in order to obtain the maximum local mean value of the region.

Then, in the common case with constant column lengths within the storeys, $K_{s j}$ 
(Eq. (13)) is replaced by a "mean" effective length factor given by

$$
\bar{K}_{s j}=\sqrt{\frac{\bar{\alpha}_{s}}{\alpha_{E j}}}=\left[\frac{P_{E j}}{P_{j}} \frac{1}{n} \sum^{n}\left[\frac{\sum \gamma P}{\sum \gamma P_{\mathrm{cr}}}\right]\right]^{1 / 2}
$$

As defined above, the summation of Eq. (15) will include 2 or more storeys when "the most flexible storey" (with the largest storey stability index) is the bottom or top storey, and 3 storeys or more otherwise. This approach may become unconservative (underestimate $\alpha_{\text {system }}$ ) in the case of "stiff beam, flexible column frames" with little interaction between the storeys (typically with $G$ factors of less than 0.8 to 1 ). In such cases, Eq. (15) with $n=1$ would be most appropriate. Also, it may become unconservative when the axial force effects $\left(\alpha_{s}\right)$ of the storey(s) adjacent to the most flexible storey are small compared to the latter.

The approach may be generalized to cover such cases by imposing the constraint

$$
\alpha_{\text {system }} \geq \lim \alpha_{s k}
$$

where $\lim \alpha_{s k}\left(\right.$ or $\lim \bar{\alpha}_{k}$ ) is a lower limit of the storey stability index of the most flexible storey, here labelled $k$. It cannot become less than the storey stability index obtained on the basis of effective length factors $(\lim K)$ calculated with $G$-factors that include the columns in the adjacent storey(s) as flexural members, with first order rotational stiffness corresponding to their far end support conditions. For rigidly connected columns the stiffness becomes $E I / L, 0$ and about $0.7 E I / L$ when the far end is fully fixed, pinned or continuous (partially restrained), respectively. The storey stability index obtained in this manner, and possibly increased by $10 \%$ or so to reflect some axial force effects in the adjacent storey(s), represents an approximate lower limit. However, as this procedure may be considered somewhat cumbersome, in particular for multibay frames, efforts to establish more simple constraints are encouraged.

\section{APPLICATION - TWO STOREY FRAMES}

Effective length results for an unbraced two storey, one bay frame are presented in Figure and 2 . The frame, shown by inserts in the same figures, is symmetrical both with respect to member properties and column axial forces. The outer boundaries of the two storeys are widely different. They are pinned and fully fixed, respectively. The results are applicable to any such frames with the given $P L$-ratio and $E I / L$-ratios. In the computations, all parameters but $E I_{2}$ (of columns in storey 2) were kept constant. Results are plotted versus $Q_{12}$ which increases with increasing $E I_{2}$ (cfr. Eq. (4)), i.e. with decreasing flexibility of column 2 relative to column 1 .

The $K$-factors labelled "isolated" are obtained from the transcendental equations for isolated free-to-sway (zero shear) columns based on the conventional $G$-factors. 


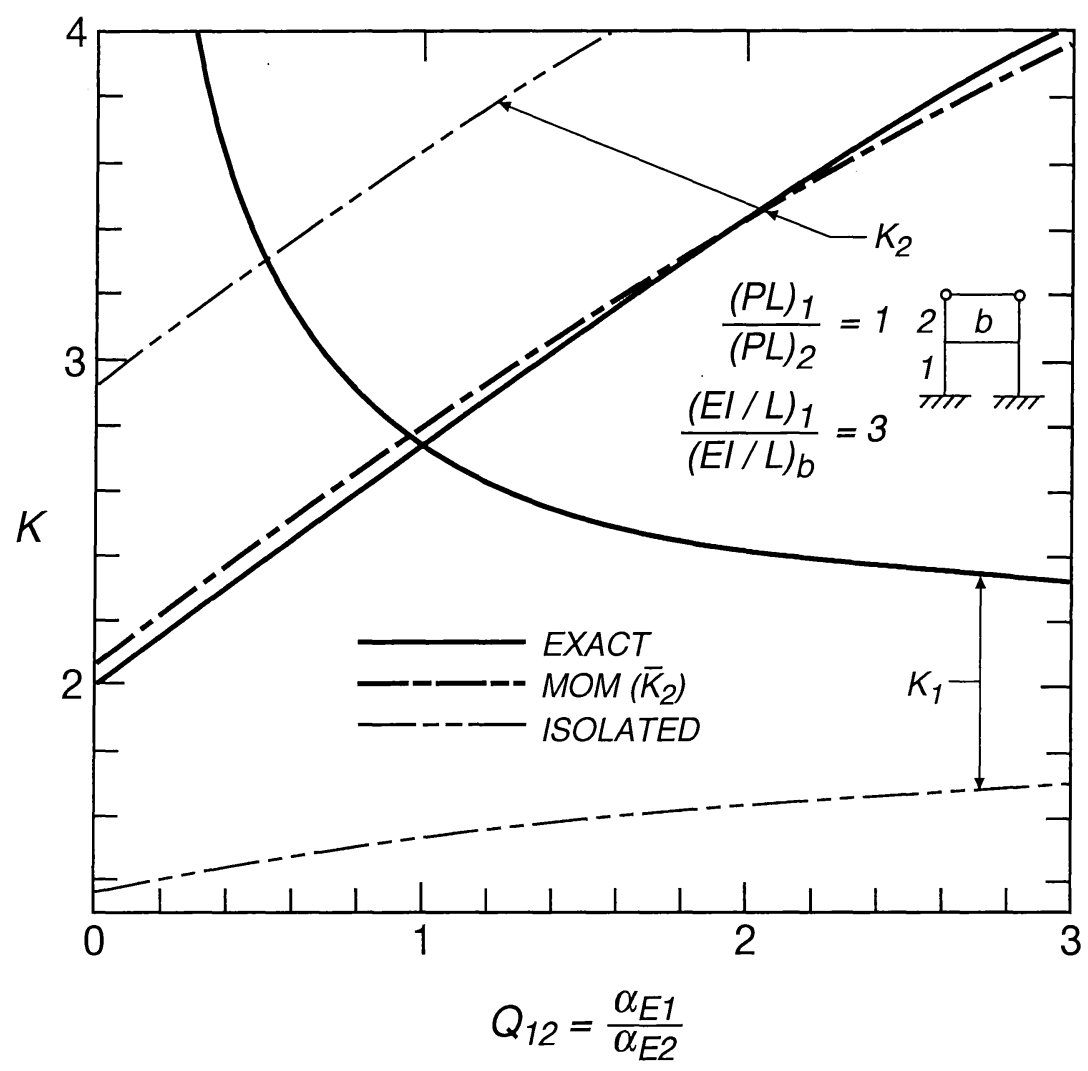

Figure 1: Effective length factors for 2-storey frame with flexible middle beam

At the middle beam joints, $m=1$ (corresponding to antisymmetrical bending). The exact results are eigenvalue solutions obtained by employing member stiffness formulations in terms of standard stability functions. The exact factors $K_{1}$ and $K_{2}$, interrelated through Eq. (3), are both shown in order to facilitate comparison with the corresponding isolated factors. Predictions by the method of means, labelled MOM and discussed below, are shown for column 2 only. Corresponding predictions for column 1 can be obtained from Eq. (3). Due to this relationship, the accuracy of the predictions will become the same for both columns (storeys).

For these frames, with identical columns in each storey, the storey indices based on $\alpha_{s i}$ become identical to those based on $\bar{\alpha}_{i}$. Furthermore, they become equal to the member indices $\alpha_{i}(i=1,2)$. Thus, $\alpha_{\text {system }}=\bar{\alpha}_{s}=\bar{\alpha}=\left(\alpha_{1}+\alpha_{2}\right) / 2$ and

$$
\bar{K}_{s 2}=\bar{K}_{2}=\sqrt{\frac{\bar{\alpha}}{\alpha_{E 2}}}=\sqrt{\frac{1}{2}\left(K_{1}^{2} Q_{12}+K_{2}^{2}\right)}
$$

The MOM predictions for the frame in Figure 1, with the more flexible middle beam, are in excellent agreement with exact results over the full range at $Q_{12}-$ values considered. As $Q_{12}$ decreases with decreasing $E I_{2}$ of column 2 relative to $E I_{1}$ of column 1, column 2 becomes increasingly restrained at the common joint. 
At $Q_{12}=0$ it is theoretically fully fixed, with an exact effective length factor of $K_{2}=2$.

For the frame in Figure 2, with a middle beam stiffness that is three times that of the frame in Figure 1, the predictions by Eq. (18) become increasingly unconservative (unsafe) as $Q_{12}$ decreases. For $Q_{12}$ less than about 2.5, the predictions follow the lower curve branching out from point $A$. As seen, they may become smaller than 2.0, which is not physically possible for a column that is pinned at one end and that is free to sway, as in this case (with no horizontal interaction between the columns of the storey). In this region, the predictions are governed by a lower limit constraint $\left(\lim \bar{K}_{2}\right)$ represented by the upper curve branching out from point $\mathrm{A}$.

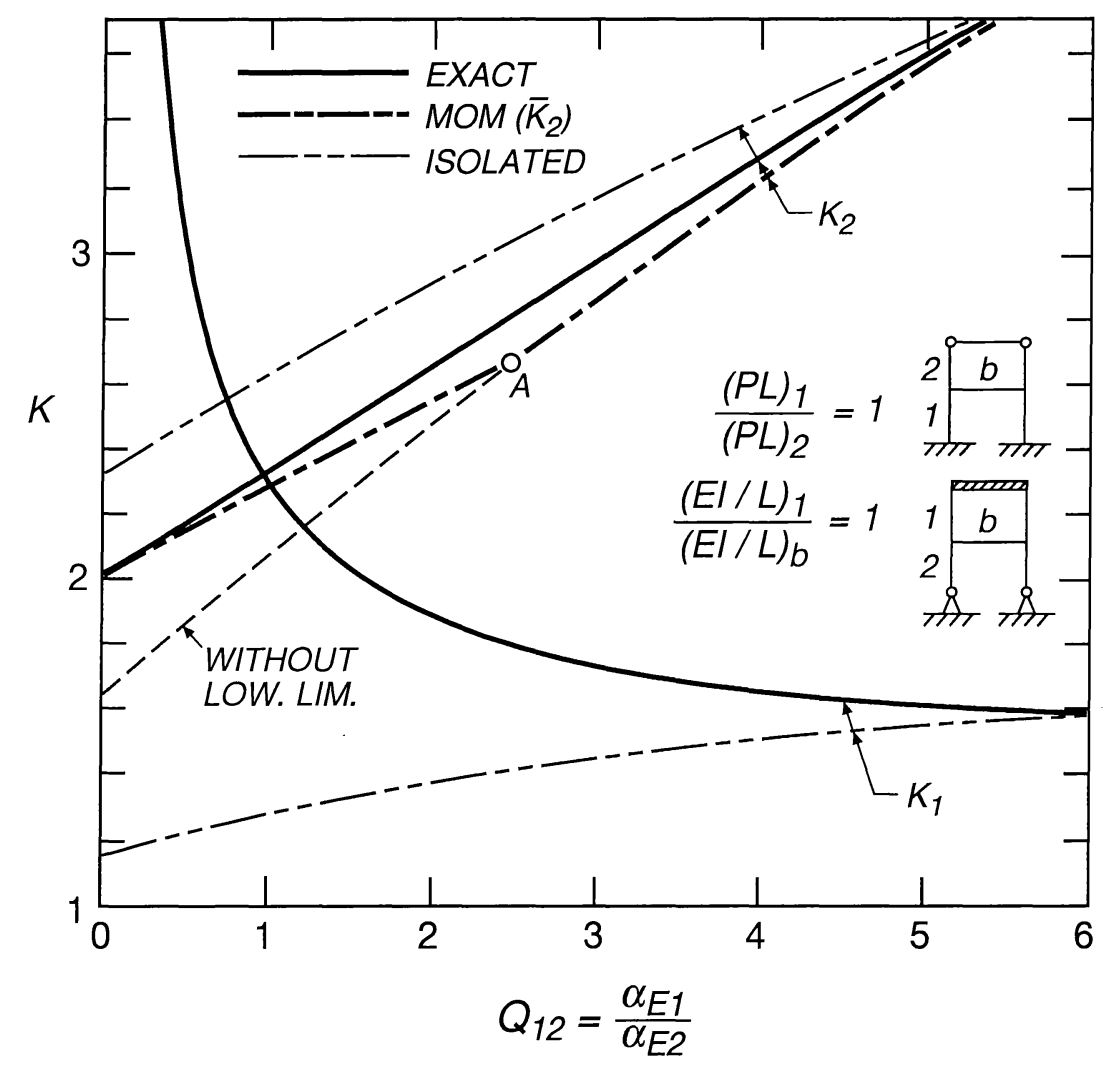

Figure 2: Effective length factors for 2-storey frame with intermediately stiff middle beam

The lower limit in Figure 2 is based on the procedure proposed in the previous section. Storey 2 (column 2 ) has the greater storey stability index. Then, by treating column 1 as a flexural member, its first order rotational stiffness becomes $(E I / L)_{1}$. Adding this to the rotational beam stiffness, the total rotational stiffness afforded to column 2 at the common joint becomes $k=6(E I / L)_{b}+(E I / L)_{1}$ and

$$
G=\frac{(E I / L)_{2}}{(E I / L)_{b}+\frac{1}{6}(E I / L)_{1}} .
$$


With this restraint at one end of column 2 and $G=\infty$ at the pinned end, the effective length factor $\lim \bar{K}_{2}$ is obtained (upper curve branching out from point A in Figure 2). With this lower limit, MOM predictions are at most $4 \%$ below exact results. As $Q_{12}$ approaches zero and the restraint assumption above becomes increasingly correct, $\lim \bar{K}_{2}$ approaches the exact solution. In a practical case, an increase of $5 \%$ or so of the limit obtained in this manner would be appropriate in order to give better estimates at practical $Q_{12}$-values.

\section{APPLICATION - MULTISTOREY FRAMES}

The applicability of the method of means is demonstrated in the following for two high-rise multistorey frames. Isolated effective length factors are computed for the columns of each storey by considering them free to sway and with beam restraints due to antisymmetrical bending ( $m=1$ in Eq. (6)).
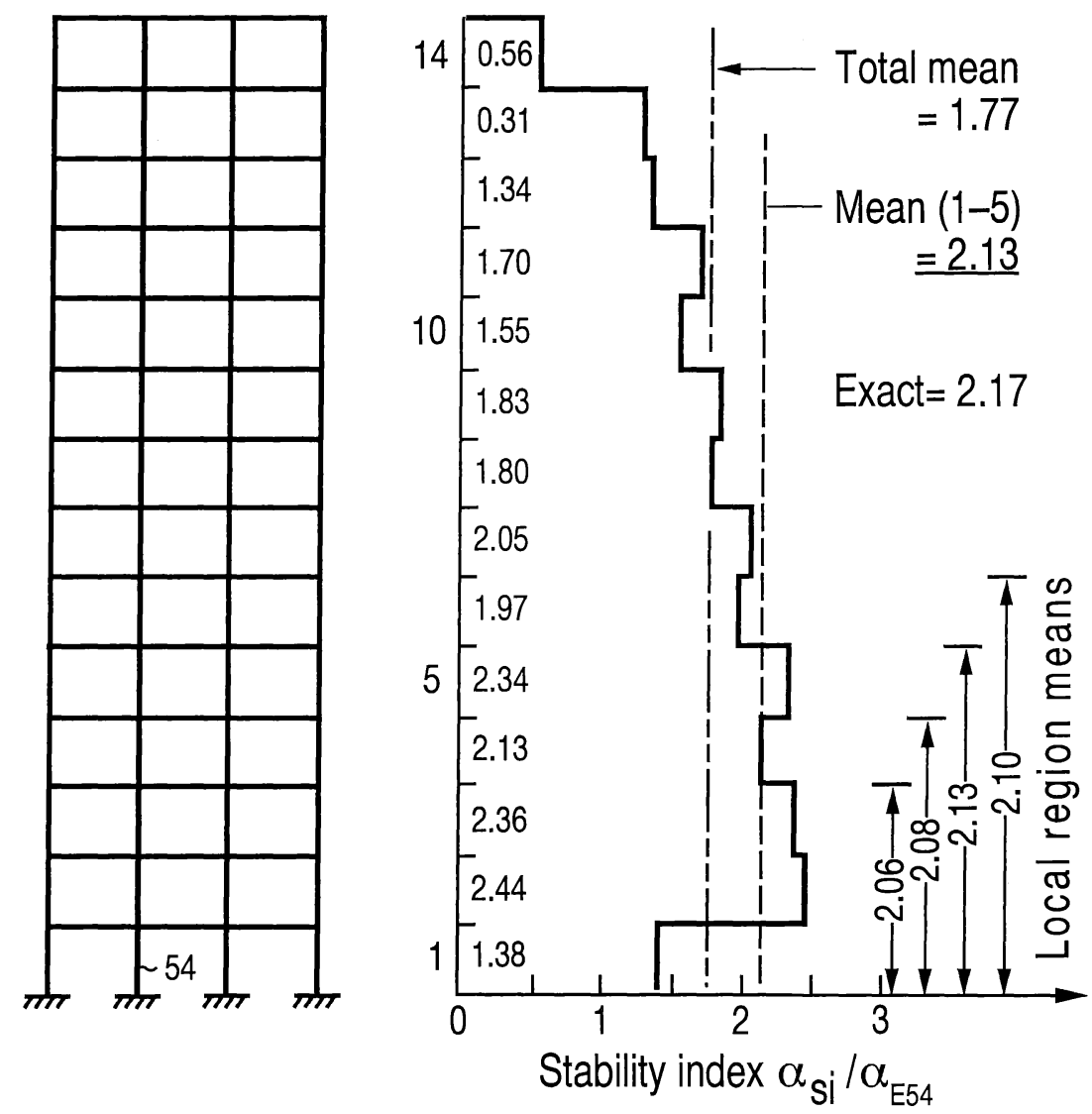

Figure 3: Stability indices for 14-storey, 3-bay frame

First, a symmetrical 14-storey, 3-bay frame is considered. It is taken from Kuhn (1976) and shown in Figure 3. It is subjected to the same symmetrical, vertical loading at each storey level. At every second storey level column stiffnesses are 
decreased. Stiffnesses in the first storey are about four times those in the top storey. Exterior columns are about 1.3 times stiffer than interior columns. Beam stiffnesses, generally smaller in the middle bay than in the exterior bays, are also decreased towards the top. For full details, see Kuhn (1976).

The $G$-factors at the exterior joints vary between about 5 at the first floor level and 3 at the seventh level, and, at the corresponding interior joints, between about 2.1 and 1.6. With such $G$-factors the frame may be classified as a "stiff column, flexible beam frame", although it is not an extreme example of such frames.

The storey stability indices $\alpha_{s i}$, with $\gamma=1$, are given in terms of $\alpha_{E}$ for column 54 (interior, first storey) by the stepped lines in Figure 3. The largest value (2.44) is found for storey 2. Adopting the approach proposed earlier, a maximum local mean for the region is obtained, as illustrated in Figure 3, as the mean of storeys $1-5$. Thus,

$$
\alpha_{\text {system }}=\bar{\alpha}_{s}(1-5)=2.13 \alpha_{E 54}
$$

which is $2 \%$ below the "exact" value (2.17) obtained from the computer solution given by Kuhn. Corresponding $K$-factors (Eq. (1)) become $1 \%$ lower than exact values. Based on the total mean (1.77), the $K$-factors would have been underestimated by $10 \%$. Almost identical results can be obtained on the basis of the storey stability indices $\bar{\alpha}_{i}$ by the method of means (2.10 instead of 2.13 and 1.74 instead of 1.77 for the local and total mean, respectively).

The second frame considered is a symmetrical 24-storey, 1-bay frame taken from Lai and MacGregor (1983). The lower 14 storeys are shown in Figure 4. The same vertical loading is applied at all storey levels. The column stiffnesses are identical within sets of eight storeys, but significantly different for the three sets. The columns of the lower storeys are considerably stiffer than the beams (18.8 times $E I_{b}$ ). Thus, the frame represents a rather extreme example of a "stiff column, flexible beam frame".

Sway magnification factors based on the storey stability indices $\alpha_{s \Delta}$ and $\alpha_{s}$, were computed and presented in diagram form by Lai et al. From an enlarged version of this diagram, magnification factors were read and stability indices rederived. The results, considered sufficiently accurate for the present purpose, are presented in Figure 4.

Regarding $\alpha_{s \Delta}, \gamma$ was taken as 1.2 in the bottom storey and as 1.05 in the others. Further, the first order lateral storey stiffnesses were obtained on the basis of a single lateral load applied at the top of the frame. This loading is different from the loading pattern proposed by Horne and reviewed above. However, the resulting maximum index of 0.45 in storey 7 is still believed to represent a reasonably correct estimate of the system stability index (cfr. Eq. (14)).

The first order bending moment diagram (due to the lateral load at the top) shows 


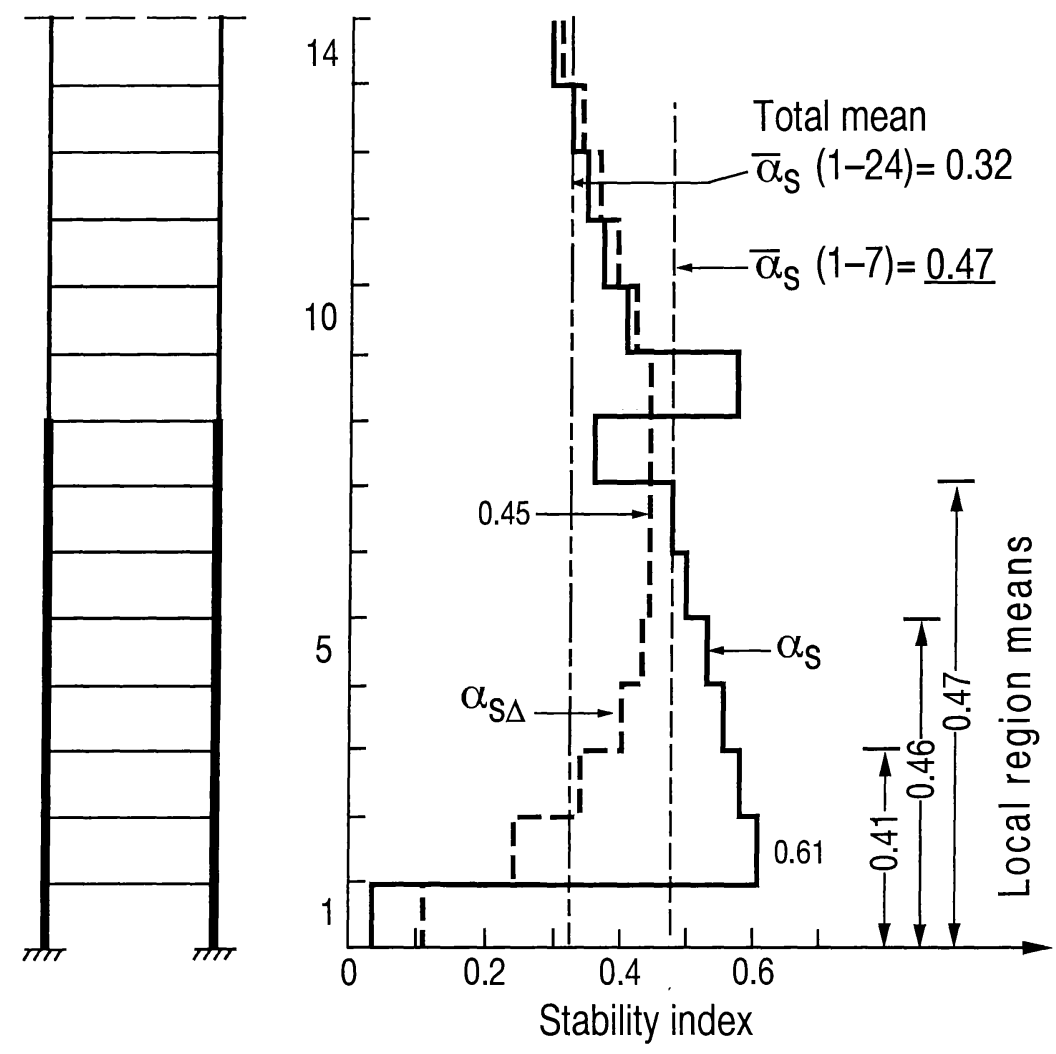

Figure 4: Stability indices for 24-storey frame

single curvature bending in the bottom 4 storeys. The significant differences in the values and variations of $\alpha_{s \Delta}$ and $\alpha_{s}$ in the lower storeys (Figure 4) are typical for regions with single curvature bending. The isolated conventional $K$-factors do not reflect the strong restraint provided by the bottom storey (fixed at the base) to the subsequent storeys. As a consequence, the storey stability indices $\alpha_{s}$ may be significantly underestimated in the bottom storey and overestimated in a number of subsequent storeys. This is seen to be the case in Figure 4, and, to a lesser extent, also in Figure 3.

The maximum local mean for the lower region centered around storey 2 (with the largest $\alpha_{s}$ ) is found to be the mean of storeys $1-7$. The means of storeys $1-6$ and 1-9 is slightly smaller. Then, from Figure 4,

$$
\alpha_{\text {system }}=\bar{\alpha}_{s}(1-7)=0.47
$$

This value corresponds well with 0.45 given by $\max \left(\alpha_{s \Delta}\right)$. Thus, the incorrect vertical interaction implied by the conventional $G$-factors and reflected in the isolated $\alpha_{s}$ predictions, is reasonably well corrected for by the method of means also in the case of this rather "extreme" frame.

Usage of the maximum $\alpha_{s}(0.61)$ would be very conservative and usage of the total mean (0.32) very unconservative (unsafe) in this case. 


\section{CONCLUDING REMARKS}

The method of means offers a simple approach for computation of effective length factors for continuous columns and frames. Its use of isolated effective length factors obtained by standard methods makes the approach particularly attractive. The same applies when used in conjunction with alternative storey based stability indices for unbraced frames. The method satisfies basic system instability principles, and does, with appropriate constraints imposed, provide critical load and effective length predictions that generally will be in excellent agreement with the results of exact system instability analyses.

\section{REFERENCES}

Australian Standard AS4100-1990. Steel structures. Standards Australia, Sydney, Australia

Eurocode 3, Design of steel structures - part 1.1: general rules and rules for buildings. (1992). European prestandard ENV, 1993-1-1, CEN, Brussels, Belgium

Hellesland, J. (1976). Approximate second order analysis of unbraced frames. Technical Report, Dr. Ing. A. Aas-Jakobsen Ltd., Oslo, Norway, 43 pp.

Hellesland, J., Simplified system instability analysis. Proc., 4th Pacific Struct. Steel Conf. (PSSC); Vol. 1, Steel Structures, 1995, Pergamon, Tarrytown, N.Y., 95-102

Hellesland, J. and Bjorhovde, R., Restraint demand factors and effective lengths of braced columns. J. Struct. Engrg., ASCE, 1996, 122(10), 1216-1224

Hellesland, J. and Bjorhovde, R., Improved frame stability analysis with effective lengths. J. Struct. Engrg., ASCE, 1996, 122(11), 1275-1283

Hellesland, J. and Frislid, A., Approximate critical load analysis of frame systems with axially compressed beams. Proc., 5th International Colloquium on Stability and Ductility of Steel Structures (SDSS), Vol. 2, 1997, T. Usami, ed., Japanese Society of Steel Construction, Nagoya University, Japan, 699-706

Horne, M.R., An approximate method for calculating the elastic critical loads of multi-storey plane frames. The Struct. Engr., 1975, 53(6), 242-248

Kuhn, G. K. (1976). An appraisal of the effective length alignment charts. Engrg. report for the degree of M. Sc. in Engineering, Arizona State University, Tempe, Arizona

Lai, S.-M.A. and MacGregor, J.G., Geometric nonlinearities in unbraced frames. J. Struct. Engrg., ASCE, 1983, 109(11), 2528-2545

LeMessurier, W.M., A practical method of second order analysis. Engrg. J., AISC, 1977, 14(2), 49-67 